\title{
INTERACTIVIDAD Y PARTICIPACIÓN EN LAS PÁGINAS WEB DE LOS PRINCIPALES DIARIOS DEPORTIVOS ESPAÑOLES
}

Jesús Díaz del Campo Lozano1: Universidad Internacional de La Rioja (UNIR) (España).

\section{jesus.diaz@unir.net}

\section{RESUMEN}

La presente investigación plantea la evaluación de la interactividad y la participación en las páginas web de los principales diarios deportivos españoles de acuerdo a los datos de audiencia correspondientes al Estudio General de Medios (EGM). En concreto, se analizan los sitios web de Marca, As, Sport y Mundo Deportivo). Para ello se emplea un modelo de análisis compuesto por 36 indicadores organizados alrededor de ocho parámetros básicos, que miden aspectos como la interacción entre el medio de comunicación y el usuario, o la adaptación del medio a las herramientas propias de la Web 2.0, entre otros. Con ello se pretende averiguar si existe una relación entre el índice de audiencia del medio y el nivel de interactividad y participación de sus sitios web. Los resultados muestran que, efectivamente, los diarios con un mayor número de lectores son los que más han adaptado su página web a las necesidades de participación e interacción que demanda el usuario.

PALABRAS CLAVE: Internet - periódicos digitales - interactividad - participación ciudadana - Web 2.0

\footnotetext{
${ }^{1}$ Jesús Díaz del Campo Lozano: Es profesor en la Facultad de Empresa y Comunicación y en la Facultad de Derecho de la Universidad Internacional de La Rioja (UNIR).Licenciado en Periodismo y doctor en Comunicación por la Universidad Complutense de Madrid.
} 
Internet - periódicos digitales - interactividad - participación ciudadana - Web 2.0

\title{
INTERACTIVITY AND PARTICIPATION IN THE MAIN SPANISH SPORT NEWSPAPERS WEBSITES
}

\begin{abstract}
This research aims to analyze the level of interactivity and citizen's participation in the websites of the major Spanish sports daily according to audience data for Estudio General de Medios (EGM). Specifically, we analyze the websites of Marca, As, Sport and Mundo Deportivo). We use an analytical model that consists of 36 indicators organized around eight basic parameters that measure aspects such as the interaction between the media and the user, or the adaptation to the Web 2.0, among others. We try to find out whether there is a relationship between the audience share and the level of interactivity and participation of the website. The results show that, indeed, the newspapers with more readers are the ones who have adapted their website to the needs of participation and interaction required by the user.
\end{abstract}

KEY WORDS: Internet - online newspapers - interactivity - citizen's participation web 2.0 


\section{INTRODUCCIÓN}

La aparición de Internet y las tecnologías de la información y la comunicación (TIC) ha alterado de manera sustancial numerosos aspectos de nuestra vida cotidiana, y la forma de relacionarnos con los medios de comunicación no ha sido una excepción. Es por ello que los medios han tratado de adaptarse a este nuevo panorama y de cubrir esas nuevas necesidades de la audiencia a través de diversas vías, mejorando la calidad de los sitios web e implementando nuevas funcionalidades para lograr una mayor interacción con el usuario. En ese sentido, el presente estudio, de carácter exploratorio, analiza la presencia de herramientas y canales que fomenten la interactividad y la participación en los sitios web de los principales diarios deportivos españoles de acuerdo a los datos del Estudio General de Medios (EGM).

Es evidente que las empresas periodísticas se han marcado como gran objetivo extraer el máximo rendimiento posible a las características del nuevo medio, Internet, una herramienta con unas capacidades que aumentan exponencialmente las posibilidades en términos de audiencias, generación de contenidos $\mathrm{y}$, muy especialmente, como una vía para lograr la rentabilidad económica (Thurman, 2008, Vujnovic et al, 2010). De ahí que si hubiese que elegir un concepto que definiera cuál ha sido el devenir todos ellos a lo largo de estos años, sin duda sería el de convergencia, entendida tal y como la definen Salaverría, García-Avilés y Masip (2010: 48):
"un proceso multidimensional que, facilitado por la implantación generalizada de las tecnologías digitales de telecomunicación, afecta al ámbito tecnológico, empresarial, profesional y editorial de los medios de comunicación, propiciando una integración de herramientas, espacios, métodos de trabajo y lenguajes anteriormente disgregados, de forma que los periodistas elaboran contenidos que se distribuyen desde múltiples plataformas, mediante los lenguajes propios de cada una".

Esa convergencia se ha producido en diferentes ámbitos del periodismo, tanto estructurales como en los procesos de producción y difusión de contenidos (Tremayne, Weiss y Alves, 2007; Paterson y Domingo, 2008, Navarro, 2009), y ha afectado a las relaciones del medio con su audiencia, con la publicidad y con los propios profesionales (Domingo et al, 2008). Y es que, en palabras de López García (2011: 91), el cambio es tan profundo que lo único que permanece es "lo básico, la esencia del periodismo -el periodismo puro y duro-, y la necesidad de los ciudadanos de disponer de información para poder moverse en la sociedad". Las principales características de este nuevo escenario, que han sido suficientemente definidas por varios autores, se refieren a la multimedialidad, la hipertextualidad, la personalización, la interactividad, la inmediatez o la posibilidad de actualización permanente, entre otras (Cabrera González, 2000; Orihuela, 2002; Masip, Díaz-Noci, Domingo, MicoSanz y Salaverría, 2010). 
Internet - periódicos digitales - interactividad - participación ciudadana - Web 2.0

El último paso en esa evolución sería el nuevo papel de los usuarios como autores o, lo que es lo mismo, el periodismo participativo en sus diferentes grados de elaboración (Díaz-Noci, 2010), con una importante novedad, se ponen en marcha espacios de participación (Bruns, 2005; Gillmor, 2004), a través de los cuales los usuarios participan en todas las fases del proceso de producción de la información, recogida, análisis y difusión (Bowman y Willis, 2003), hasta llegar a aportar contenidos propios (Bardoel, 1996; Deuze, 1999).

Ahondando en ese tipo de cuestiones en el caso concreto de España, el estudio La Prensa: digital vs papel, elaborado por la Asociación para la Investigación de Medios de Comunicación (AIMC) y presentado en 2011, mostraba entre sus conclusiones las siguientes:

1. Un $32 \%$ de los internautas afirma que lee diaria y simultáneamente prensa digital y en papel. La cifra aumenta hasta el 59\% hablando del último mes.

2. Entre los motivos para leer los diarios en soporte tradicional destacan los aspectos relacionados con el contenido y el análisis, mientras que la actualidad es el principal factor que lleva a consumir la prensa digital.

3. El promedio de diarios online consultados habitualmente es de 2,9 títulos diferentes, cifra superior a la diarios offline, que se sitúa en 2,1.

4. La gratuidad de muchos medios y la facilidad para consultar la hemeroteca son dos de los factores más apuntados por los lectores exclusivos de prensa online.

En cuanto a otros estudios llevados a cabo hasta la fecha en nuestro país, de los referidos a la televisión destaca el de Meso, Palomo y Ganzábal (2009), que analiza la presencia de elementos propios del periodismo participativo en seis cadenas, Rtve.es; Antena3.com, Telecinco.es, Plus.es, LaSexta.com y Cuatro.com. Una de sus conclusiones más relevantes es que las cadenas nacionales españolas suponían en ese momento una especie de laboratorio de pruebas. Asimismo, los autores destacaban que a finales de 2008 las seis cadenas analizadas ponían a disposición del usuario un completo de opciones de participación tras haber incorporado diversas mejoras a sus páginas web con el objetivo de mejorar su usabilidad y dinamismo y de hacerlas más participativas.

Por lo que respecta a la radio, uno de los estudios más recientes (Peña, 2012), que analizaba el uso de las herramientas de la web 2.0 por parte de las emisoras españolas más importantes, remarcaba el contraste entre las enormes posibilidades que ofrece la red y el aprovechamiento limitado que de ellas realizan las emisoras, algo que se materializaba en dos ámbitos:

- El empleo de herramientas como el correo electrónico, las redes sociales, los blogs, los foros o los chats, todavía poco extendido, hasta el punto de que tan sólo algo más de la mitad de espacios radiofónicos diarios hacía uso de ellos.

- La participación de los oyentes, deseosos de adquirir un mayor protagonismo, de realizar aportaciones al medio y, en general, de aumentar la interactividad, frente a un medio que se rige todavía por el esquema tradicional. 
En cuanto a la prensa escrita, cabe mencionar trabajos como los de Salaverría, Cores, Díaz-Noci, Meso y Larrondo, 2004, referido a los medios vascos y navarros, o el de Salaverría et al (2005), en el que se estudiaban las ediciones digitales de algunas de las principales diarios de información general en nuestro país. La idea de experimentación permanente y de proceso a medio concluir era una de las notas dominantes, al igual que sucedía en el caso de la televisión. En la misma línea se situaban las conclusiones de Cantalapiedra et al (2010) en su análisis de los diarios vascos en Internet; o el estudio referido a los cibermedios gallegos (López García, 2011), en el que se hablaba de experimentación "sin muchas prisas" y "sin asumir muchos riesgos". Por su parte, Martínez (2008) en una investigación referida a diarios exclusivamente digitales iba más allá al hablar de una predisposición negativa de estos medios a la hora de incorporar herramientas interactivas.

Por tanto, se observa que el desaprovechamiento de las posibilidades que ofrece Internet o la inclusión de las mismas de una manera todavía muy incipiente o en un camino todavía por terminar es una constante que se repite de manera casi unánime en las conclusiones de los distintos estudios que se han llevado a cabo, con independencia del medio analizado.

Sin embargo, para completar el análisis faltaría un sector fundamental al menos en cuanto a difusión: cuatro de los siete diarios más leídos en España, según los datos del EGM², son deportivos. Sí que se han analizado aspectos muy específicos de los sitios web de algunos de ellos y con ocasión de la celebración de eventos muy concretos (Barredo Ibáñez y Oller Alonso, 2012), pero no existe hasta la fecha un estudio sistemático que evalúe las páginas web de los principales diarios de este sector tan representativo en la prensa española.

Por ello, la presente investigación pretende realizar una primera exploración de ese sector, centrándose en el análisis de la puesta en marcha de herramientas que fomenten la participación y la interactividad con la audiencia, y tomando como punto de partida el marco teórico establecido, se marca los siguientes objetivos:

1. Analizar si los principales diarios deportivos españoles han llevado a cabo una adaptación adecuada al entorno de Internet y determinar cuáles son las posibilidades que, en términos de interactividad y participación, ofrecen sus respectivos sitios web.

2. Establecer qué parámetros son los que reflejan unos mayores niveles de adaptación y qué otros son susceptibles de mejora.

3. Comprobar si existe coincidencia entre el índice de audiencia de un diario deportivo y el nivel de interactividad y participación de su sitio web.

\footnotetext{
${ }^{2}$ Así se refleja en el Estudio General de Medios con los datos referidos al año móvil abril 2012 - marzo 2013.
} 
Internet - periódicos digitales - interactividad - participación ciudadana - Web 2.0

Para ello se ha analizado una muestra conformada por los cuatro diarios deportivos que aparecen entre la relación de los 50 diarios españoles con mayor número de lectores según los datos del Estudio General de Medios (EGM) referido al año móvil abril 2012-marzo 2013 (Tabla 1).

Tabla 1. Diarios deportivos seleccionados para el estudio

\begin{tabular}{|l|c|}
\hline Cadena & Lectores/día en miles \\
\hline Marca & 2.870 \\
\hline As & 1.426 \\
\hline Sport & 678 \\
\hline Mundo Deportivo & 672 \\
\hline
\end{tabular}

Fuente: Elaboración propia a partir de datos del EGM

De este modo, se han evaluado los sitios web de los diarios que se enumeran a continuación: Marca (marca.com), As (as.com), Sport (sport.es), y Mundo Deportivo (mundodeportivo.com).

\section{METODOLOGÍA}

El método utilizado para evaluar el grado de interactividad de los sitios web de los cinco diarios deportivos estudiados es el propuesto por el profesor Lluís Codina, recogido en diversos textos, muchos de los cuales pueden consultarse en su página web, www.lluiscodina.com, y una de cuyas últimas versiones se ha publicado recientemente (Rodríguez Martínez, Codina y Pedraza-Jiménez, 2012).

Esta herramienta, en cuya elaboración se han tenido en cuenta diversos trabajos y modelos de análisis (Zamith, 2008; Guallar y Abadal, 2009; Palacios y Díaz-Noci, 2009; Rodríguez Martínez, Codina y Pedraza-Jiménez, 2010) pone especial énfasis en la interacción y la personalización, pero sin dejar de lado otros aspectos relacionados con la usabilidad y la arquitectura de la información (Rodríguez Martínez, Codina y Pedraza-Jiménez, 2012, 62).

Este método de evaluación, surgido del análisis de una multitud de sitios web entre los que se encontraban los de diarios internacionales y nacionales, canales de televisión, blogs elaborados por periodistas y sitios web de periodismo ciudadano, entre otros (Rodríguez Martínez, Codina y Pedraza-Jiménez, 2012, 63) se basa en el establecimiento de dimensiones, parámetros e indicadores que permitan examinar sitios web de medios de comunicación y conocer si han hecho o no una adaptación adecuada al entorno de la web (Díaz-Noci et al, 2009) .

El modelo de análisis, que ya ha sido utilizado en otros estudios similares (por ejemplo Salaverría, Cores, Díaz-Noci, Meso y Larrondo, 2004), se compone de seis dimensiones: cooperación, participación, creación de contenido, acceso al contenido, socialización y comunicación; mientras que emplea ocho parámetros: interacción usuario-medio de comunicación; publicación de contenidos creados por los usuarios; registro del usuario; acceso a la información; canal de contenido personalizado; el 
medio ofrece distintas versiones de su información; empleo de herramientas de la Web 2.0; y plataformas de la Web 2.0 en las que tiene presencia el medio de comunicación.

A su vez, cada parámetro agrupa varios indicadores, hasta un total de 36 :

1. Parámetro 1: interacción medio de comunicación-usuario.

1.1. Comunicación con el autor de la noticia.

1.2. Contacto con la redacción del medio de comunicación.

1.3. Comentar noticias publicadas por el medio de comunicación.

1.4. Votación de noticias publicadas por el medio de comunicación.

1.5. Comentar entradas publicadas en los blogs del medio de comunicación.

1.6. El usuario puede modificar o corregir contenido publicado por el medio.

2. Parámetro 2: publicación de contenidos creados por los usuarios.

2.1. Creación de blogs por los usuarios.

2.2. Publicación de textos escritos por los usuarios.

2.3. Publicación de fotos tomadas por los usuarios.

2.4. Publicación de vídeos realizados por los usuarios.

2.5. Sección exclusiva para contenido creado por los usuarios.

3. Parámetro 3: registro del usuario.

3.1. Registro por parte del usuario en el medio.

3.2. Contacto con otros usuarios registrados.

4. Parámetro 4: acceso a la información.

4.1. Acceso a la información a través de la portada.

4.2. Acceso a la información a través de secciones.

4.3. Acceso a la información a través de noticias relacionadas.

4.4. Acceso a la información a través del buscador.

4.5. Acceso a la información a través del mapa web.

4.6. Acceso a la información a través de la recomendación de los usuarios.

4.7. Acceso a la información a través de plataformas externas de la Web 2.0.

5. Parámetro 5: personalización de la información.

5.1. Adaptación de la interfaz del sitio web del medio de comunicación en función de los contenidos de interés para el usuario.

5.2. Sindicación de contenidos del medio de comunicación a través del móvil o correo electrónico.

5.3. Suscripción de alertas o boletín electrónico.

6. Parámetro 6: el medio ofrece distintas versiones de su información.

6.1. Versión impresa del medio.

6.2. Versión global.

6.3. Versión actualizada de forma constante.

6.4. Versión impresa adaptada a la Web 2.0.

7. Parámetro 7: empleo de herramientas de la Web 2.0.

7.1. Compartir información con otros usuarios.

7.2. Blogs vinculados al medio de comunicación.

8. Parámetro 8: plataformas de la Web 2.0 en las que tiene presencia el medio de comunicación. 
Internet - periódicos digitales - interactividad - participación ciudadana - Web 2.0

8.1. Presencia del medio de comunicación en plataformas audiovisuales.

8.2. Presencia del medio de comunicación en plataformas de imágenes.

8.3. Empleo de redes sociales propias.

8.4. Presencia del medio de comunicación en redes sociales profesionales externas.

8.5. Presencia del medio de comunicación en redes sociales de amistad.

8.6. Presencia del medio de comunicación en plataformas de microblogging.

8.7. Vinculación entre el sitio web del medio de comunicación y las plataformas sociales.

El análisis de las páginas web se realizó durante la semana del 7 al 11 de octubre de 2013. A diferencia del método original (Rodríguez-Martínez, Codina y PedrazaJiménez, 2012), que proponía en algunos indicadores concretos una puntuación de 0 a 3 , se ha considerado más objetivo que cada pregunta sólo pueda ser puntuada con un 0 o un 1 , respondiendo a la ausencia o presencia de cada característica respectivamente y añadiendo una puntuación general para medir el grado de interactividad.

\section{ANÁLISIS Y DISCUSIÓN}

\subsection{Interacción}

La interacción entre un medio de comunicación y sus usuarios consiste en establecer una relación comunicativa en la que ambas partes interactúan, un hecho que implica un cambio muy significativo en la naturaleza del proceso de producción de la información: el usuario adquiere un protagonismo mucho mayor y participa de manera más activa en el proceso. Pues bien, los resultados obtenidos en este apartado (Tabla 2) revelan que:

Tabla 2: Interacción medio de comunicación-usuario

\begin{tabular}{|l|c|c|c|c|}
\hline Indicador & MARAA & MPNO \\
\hline Comunicar autor & & & & \\
\hline Contactar redacción & $\mathrm{X}$ & $\mathrm{X}$ & $\mathrm{X}$ & $\mathrm{X}$ \\
\hline Comentar noticias & $\mathrm{X}$ & $\mathrm{X}$ & $\mathrm{X}$ & $\mathrm{X}$ \\
\hline Votar noticias & $\mathrm{X}$ & & $\mathrm{X}$ & \\
\hline Comentar blogs & $\mathrm{X}$ & $\mathrm{X}$ & $\mathrm{X}$ & $\mathrm{X}$ \\
\hline Modificar contenido & & & & \\
\hline
\end{tabular}

Fuente: Elaboración propia

1. Ninguno de los cuatro diarios analizados cumple todos los indicadores recogidos en el apartado.

2. El grado de cumplimiento es muy similar entre todos ellos, con una única diferencia reseñable: la posibilidad de votar la noticia, presente en Marca y Sport, pero no en As y Mundo Deportivo.

3. Sin embargo, el nivel de cumplimiento de los distintos indicadores es muy desigual, ya que mientras la posibilidad de contactar con la redacción y de comentar las noticias y los contenidos de los blogs están presentes en las 
páginas web de los cuatro periódicos, ninguno de ellos permite contactar de forma directa con el autor de la noticia ni tampoco modificar el contenido de la misma. En este sentido, llama la atención el hecho de que los medios analizados hayan apostado de forma más clara por la posibilidad de que el usuario comente sus contenidos que por la opción de votarlos y/o calificarlos.

\subsection{Publicación de contenidos}

Este parámetro constituye en buena medida una continuación del anterior, por cuanto supone dar un paso más en la búsqueda del mayor protagonismo de los lectores, lo que se traduce en que más allá de participar en el proceso comunicativo -a través de los foros, comentarios, chats, blogs, encuestas u otras herramienta similares- producen sus propios contenidos, ya sea en forma de textos, vídeos o fotografías, y adquieren el rol de emisor en ese proceso.

Sin embargo, los resultados (Tabla 3) muestran que el nivel de cumplimiento de este parámetro es sensiblemente menor que en el caso de la interacción por el usuario, ya que sólo uno de los medios analizados, Marca, cumple más de la mitad de los indicadores, al permitir a sus usuarios enviar textos, vídeos y/o fotografías para publicar. Sorprende quizá por ello que este diario no haya habilitado la opción de que sus lectores creen sus propios blogs, algo que sí pueden hacer los usuarios de As y de Mundo Deportivo.

Por último, cabe destacar el hecho de que ninguno de los diarios haya creado una sección destinada a la publicación del contenido generado por los usuarios, una opción todavía no muy común en la prensa española, pero que ya está presente, por ejemplo, en la página web del diario La Vanguardia (Rodríguez-Martínez, Codina y Pedraza-Jiménez, 2012: 72-73).

Tabla 3: Publicación de contenidos creados por el usuario

\begin{tabular}{|l|c|c|c|c|}
\hline Indicador & MARAA & SPER & SPNDO \\
\hline Crear blogs & & $\mathrm{X}$ & & $\mathrm{X}$ \\
\hline Publicar textos & $\mathrm{X}$ & & & \\
\hline Publicar fotografías & $\mathrm{X}$ & & & \\
\hline Publicar vídeos & $\mathrm{X}$ & & & \\
\hline Sección exclusiva & & & & \\
\hline
\end{tabular}

Fuente: Elaboración propia

\subsection{Registro del usuario}

Muchos medios de comunicación exigen al usuario que se registre para poder participar activamente: los comentarios de noticias o contenidos en blogs, la recomendación de algún contenido, o el envío de textos, fotografías o vídeos son algunas de las opciones de interactividad que requieren de este paso previo por parte del usuario. En ocasiones, el registro también posibilita que el usuario pueda relacionarse con otros miembros de la audiencia a través de comunidades virtuales 
Internet - periódicos digitales - interactividad - participación ciudadana - Web 2.0

como foros o chats, creadas en el sitio web con el objetivo principal de que el público pueda interactuar.

Pues bien, los principales diarios deportivos españoles cumplen con los indicadores de este parámetro de una forma muy desigual (Tabla 4), ya que si bien todos ellos han puesto en marcha el primer paso, es decir, el registro previo, por el contrario sólo Marca permite que cualquier usuario contacte con otros usuarios del sitio web.

No obstante, cabe señalar que el abanico de opciones que se le abre al usuario a partir de dar el paso del requisito previo varía de un medio a otro, algo que por otra parte es fácil puede deducir a partir de los resultados obtenidos en otras partes de este análisis.

Tabla 4: Registro del usuario

\begin{tabular}{|l|c|c|c|c|}
\hline Indicador & MARAA & SORT & SPORT & XNDO \\
\hline Registro previo & $\mathrm{X}$ & $\mathrm{X}$ & $\mathrm{X}$ & $\mathrm{X}$ \\
\hline Contacto con otros usuarios & $\mathrm{X}$ & & & \\
\hline
\end{tabular}

Fuente: Elaboración propia

\subsection{Acceso a la información}

Cualquier sitio web almacena una cantidad ingente de información, y los medios de comunicación no son una excepción. Es por ello que su gestión y clasificación es una tarea básica a la hora de facilitar al máximo las búsquedas de los usuarios, de modo que se trata de herramientas quizá no muy complejas en cuanto a su configuración pero con una importancia capital a la hora de orientar al público.

Los diarios analizados parecen ser conscientes de esa realidad, pues este parámetro es uno de los que ha registrado un mayor grado de cumplimiento. Asimismo es curioso que los resultados sean idénticos para los cuatro periódicos, que coinciden en cumplir seis de los siete indicadores analizados (Tabla 5).

En este sentido, las posibilidades de los usuarios de los sitios web estudiados para acceder a la información son muy diversas, y entre ellas se encuentran dos opciones muy interesantes desde el punto de vista de los contenidos: por un lado, el acceso a través de noticias relacionadas, es decir, cuando un lector consulta una determinada información encuentra enlaces a otros contenidos de temática similar que le pueden servir para contextualizar la información original. Por otro lado, todos los medios de comunicación permiten a sus usuarios en Facebook acceder a las informaciones de las cuales publican extractos en sus perfiles en esta red social.

El contrapunto a esta tendencia es el mapa web, un recurso bastante útil ya que constituye un índice de las distintas páginas del sitio a las que pueden acceder los usuarios, pero con el que no cuenta ninguno de los medios analizados. 


\begin{tabular}{|l|c|c|c|c|}
\hline Indicador & MARRA & SO & SPORT & MUNDO \\
\hline Acceso portada & $\mathrm{X}$ & $\mathrm{X}$ & $\mathrm{X}$ & $\mathrm{X}$ \\
\hline Acceso secciones & $\mathrm{X}$ & $\mathrm{X}$ & $\mathrm{X}$ & $\mathrm{X}$ \\
\hline Acceso noticias relacionadas & $\mathrm{X}$ & $\mathrm{X}$ & $\mathrm{X}$ & $\mathrm{X}$ \\
\hline Acceso buscador & $\mathrm{X}$ & $\mathrm{X}$ & $\mathrm{X}$ & $\mathrm{X}$ \\
\hline Acceso mapa web & & & & \\
\hline Acceso recomendación & $\mathrm{X}$ & $\mathrm{X}$ & $\mathrm{X}$ & $\mathrm{X}$ \\
\hline Acceso web 2.0 & $\mathrm{X}$ & $\mathrm{X}$ & $\mathrm{X}$ & $\mathrm{X}$ \\
\hline
\end{tabular}

Fuente: Elaboración propia

\subsection{Personalización del contenido}

La facultad del usuario para personalizar el contenido de una página web en función de sus intereses alcanza una de sus máximas expresiones cuando tiene la posibilidad de modificar el aspecto, las características y los contenidos del sitio, de modo que cada vez que lo visita como usuario registrado accede directamente a su página personalizada, tal y como él mismo la ha rediseñado.

En definitiva, la audiencia disfruta de un abanico de posibilidades mucho más variado, en lo que supone, en términos de personalización del contenido, un significativo paso adelante respecto a otras de las opciones que de modo más habitual se pueden encontrar en las páginas web de los medios de comunicación en los últimos tiempos: los servicios "a la carta" o "bajo demanda", es decir, la posibilidad de que el usuario escuche cualquier programa a cualquier hora del día y sin tener que tener encendido su aparato receptor de radio en el momento concreto de la emisión.

La adaptación de la interfaz según los gustos o preferencias de la audiencia implica ir mucho más allá, materializando la posibilidad de crear una página distinta para usuario, algo que, de acuerdo al análisis realizado, ninguno de los diarios analizados ofrece. (Tabla 6).

Por su parte, la sindicación de contenidos y las alertas y boletines sí que están presentes en todos los medios estudiados, que parecen ser conscientes de la necesidad de llegar con sus contenidos a todo tipo de dispositivos móviles y, en consecuencia, a una audiencia potencial mayor.

Tabla 6: Personalización del contenido

\begin{tabular}{|l|c|c|c|c|}
\hline Indicador & MARAA & SPRT & MUNO \\
\hline Adaptación interfaz & & & & \\
\hline Sindicación contenidos & $\mathrm{X}$ & $\mathrm{X}$ & $\mathrm{X}$ & $\mathrm{X}$ \\
\hline Alertas/boletines & $\mathrm{X}$ & $\mathrm{X}$ & $\mathrm{X}$ & $\mathrm{X}$ \\
\hline
\end{tabular}

Fuente: Elaboración propia 
Internet - periódicos digitales - interactividad - participación ciudadana - Web 2.0

\subsection{Distintas versiones de la información}

Los resultados obtenidos en este apartado por los diarios analizados se pueden resumir en dos elementos principales: la similitud de los datos de los cuatro medios, que coinciden plenamente en el cumplimiento de los diferentes indicadores; y por otro lado el desigual grado de cumplimiento de dichos indicadores (Tabla 7).

De este modo, los cuatro diarios coinciden en permitir que el usuario lea a través de sus sitios web la información tal y como aparece en su versión impresa y asimismo actualiza sus informaciones de manera constante. Por el contrario, ninguno de ellos cuenta con una versión global, esto es, una versión en la que se facilita el acceso mediante enlaces a otros medios, de modo que el público puede comprobar cómo han sido tratadas las informaciones en esos otros medios. Y lo mismo ocurre con la versión impresa adaptada a la web, es decir, una versión impresa que se actualiza al mismo tiempo que la versión de la web.

Tabla 7: Distintas versiones de la información

\begin{tabular}{|l|c|c|c|c|}
\hline Indicador & MARAA & SPOR & SWNDO \\
\hline Versión impresa del medio & $\mathrm{X}$ & $\mathrm{X}$ & $\mathrm{X}$ & $\mathrm{X}$ \\
\hline Versión global & & & & \\
\hline Actualización constante & $\mathrm{X}$ & $\mathrm{X}$ & $\mathrm{X}$ & $\mathrm{X}$ \\
\hline Versión impresa adaptada a la Web 2.0 & & & & \\
\hline
\end{tabular}

Fuente: Elaboración propia

\subsection{Empleo de herramientas de la web 2.0}

Uno de los principales cambios que ha experimentado Internet desde su aparición ha sido el paso de la llamada web estática o 1.0 a la denominada web social 2.0. La posibilidad de compartir contenidos y la de poner en marcha blogs son dos de los ejemplos más claros de este nuevo enfoque y los principales deportivos españoles realizan una apuesta muy decidida en esa línea, como lo demuestra el hecho de que éste sea el único parámetro (Tabla 8) en el que se cumplen el 100\% de los indicadores.

Obviamente, habría que matizar que las características de los blogs de los cuatro sitios web analizados son distintas, y van desde los que se centran en una sección o deporte concreto, hasta los de carácter más personal, protagonizados por redactores o colaboradores del medio. Lo que sí resulta evidente es que se trata de espacios que en muchos casos generan bastante participación a través de los comentarios que realizan los usuarios. 
Tabla 8: Empleo de herramientas de la Web 2.0.

\begin{tabular}{|l|c|c|c|c|}
\hline & MARAA & SORT & SPORT & MWNOD \\
\hline Compartir información & $\mathrm{X}$ & $\mathrm{X}$ & $\mathrm{X}$ & $\mathrm{X}$ \\
\hline Blogs & $\mathrm{X}$ & $\mathrm{X}$ & $\mathrm{X}$ & $\mathrm{X}$ \\
\hline
\end{tabular}

Fuente: Elaboración propia

\subsection{Presencia del medio en plataformas de la web 2.0}

Las redes sociales se han convertido en poco tiempo en herramientas cotidianas para la mayoría de internautas de todo el mundo, y España es uno de los países en los que esa tendencia alcanza cotas más altas, como reflejaba el informe del Observatorio Nacional de las Telecomunicaciones y de la Sociedad de la Información (2012), que situaba a España como el tercer país del mundo -y el primero de la Unión Europeaen uso de las redes sociales en Internet. Asimismo, según el informe, Facebook es la más usada, mientras que Youtube y Twitter también obtienen cifras importantes.

Los diarios deportivos de nuestro país parecen ser conscientes de estos datos, por cuanto todos ellos tienen cuentas en Facebook y Twitter (Tabla 8), cuyos iconos incluyen también en las respectivas portadas de sus sitios web. No existe tanta unanimidad en el caso de Youtube, donde se observa una clara diferencia entre los dos diarios editados en Madrid, As y Marca, que sí cuentan con canal propio, al contrario de lo que ocurre con los medios catalanes, Sport y Mundo Deportivo.

Por otro lado, los resultados son radicalmente diferentes en el caso de las redes sociales más profesionales y/o especializadas, como Flickr o LinkedIn, en las que hasta ahora no han considerado oportuno estar presentes los medios analizados.

Por lo demás, con la excepción señalada de la presencia en Youtube, los resultados obtenidos en este apartado son también bastante similares para los cuatro diarios deportivos.

Tabla 9: Presencia del medio en plataformas de la Web 2.0.

\begin{tabular}{|l|c|c|c|c|}
\hline Indicador & MARAA & SPNDO \\
\hline Youtube & $\mathrm{X}$ & $\mathrm{X}$ & & \\
\hline Flickr & & & & \\
\hline RR. SS. propias & & & & \\
\hline LinkedIn & & & & \\
\hline Facebook & $\mathrm{X}$ & $\mathrm{X}$ & $\mathrm{X}$ & $\mathrm{X}$ \\
\hline Twitter & $\mathrm{X}$ & $\mathrm{X}$ & $\mathrm{X}$ & $\mathrm{X}$ \\
\hline Vínculos & $\mathrm{X}$ & $\mathrm{X}$ & $\mathrm{X}$ & $\mathrm{X}$ \\
\hline
\end{tabular}

Fuente: Elaboración propia 
Internet - periódicos digitales - interactividad - participación ciudadana - Web 2.0

\section{CONCLUSIONES}

Los resultados de esta investigación permiten dar respuesta a los tres objetivos planteados al inicio de este trabajo:

1. Los principales diarios deportivos españoles están en pleno proceso de adaptación de sus páginas web, un proceso sensiblemente más avanzado en el caso de Marca, que alcanza casi un $70 \%$ de cumplimiento de los indicadores analizados, que en los otros periódicos estudiados, los cuales no obstante se sitúan todos ellos en porcentajes superiores al $50 \%$.

2. El empleo de herramientas propias de la web 2.0 y el acceso a la información por parte del usuario son los dos parámetros con un mayor grado de cumplimiento, que alcanza, en el primer caso, el 100\%. No obstante, puede decirse que el grado de implantación es bastante aceptable en la mayoría de los casos, pues no hay ningún parámetro, a excepción de la creación de contenidos por parte del usuario, que "suspenda", esto es, que obtenga en los resultados globales (Tabla 10) un porcentaje menor que el $50 \%$.

3. Existe un alto grado de coincidencia entre el grado de interactividad y participación de los sitios web y el índice de audiencia de cada uno de los diarios deportivos, ya que Marca es el que obtiene los mejores resultados, seguido de As. Mientras, Mundo Deportivo y Sport, cuyas cifras de audiencia son muy similares, alcanzan idéntico resultado. Si bien los resultados de todos ellos son similares, las diferencias se dan de manera especial en dos de los parámetros (Tabla 10), como son la creación de contenidos por parte del usuario y el registro previo, en los que Marca alcanza un nivel de implementación superior al resto de diarios estudiados.

En definitiva, los diarios deportivos españoles, y muy en especial Marca, parecen haber apostado por este tipo de herramientas interactivas de manera más decidida que por ejemplo las grandes cadenas radiofónicas (Peña, 2012) y las posibilidades para sus lectores en términos de participación e interactividad son muy amplias y diversas, sin llegar todavía a ser las máximas posibles.

Tabla 10: Resultados totales

\begin{tabular}{|l|c|c|c|c|c|c|}
\hline & MARAA & Total & \% \\
\hline Interacción usuario & 4 & 3 & 4 & 3 & 14 & 58,3 \\
\hline Contenidos creados por usuario & 3 & 1 & 0 & 1 & 5 & 25,0 \\
\hline Registro & 2 & 1 & 1 & 1 & 5 & 62,5 \\
\hline Acceso & 6 & 6 & 6 & 6 & 24 & 85,7 \\
\hline Personalización & 2 & 2 & 2 & 2 & 8 & 66,7 \\
\hline Distintas versiones & 2 & 2 & 2 & 2 & 8 & 50,0 \\
\hline Empleo herramientas web 2.0 & 2 & 2 & 2 & 2 & 8 & 100,0 \\
\hline Plataformas & 4 & 4 & 3 & 3 & 14 & 50,0 \\
\hline Total & 25 & 21 & 20 & 20 & 86 & 59,7 \\
\hline \% & 69,4 & 58,3 & 55,5 & 55,5 & 59,7 & \\
\hline
\end{tabular}

Fuente: Elaboración propia 
Esos avances resultan más evidentes en todo lo relacionado con la presencia del medio de comunicación en plataformas de la web 2.0 y a la hora de facilitar el acceso a la información al lector, pero son también notorios en el resto de aspectos analizados, a excepción de la creación de contenidos por parte del usuario, algo que quizá pueda marcar el camino a seguir en un futuro, hasta llegar a concretar lo que Bowman y Willis (2003) denominaron modelo de medio abierto exclusivo.

En todo caso, los diarios deportivos españoles, al igual que el resto de medios, deberán seguir atentamente las innovaciones que a buen seguro se irán produciendo ya que es posible que el camino hacia la convergencia tecnológica nunca culmine debido precisamente a que las novedades se suceden a tal velocidad que la puesta al día es una tarea prácticamente inalcanzable.

\section{AGRADECIMIENTOS}

Investigación llevada a cabo por el proyecto "Los medios de comunicación en Facebook: presencia, análisis y tendencias", financiado por el Plan Propio de Investigación 2011-2013 de UNIR. (http:/ / research.unir.net)

\section{REFERENCIAS}

Asociación para la Investigación en Medios de Comunicación (AIMC) (2011). La Prensa: Digital vs. papel. Consultado el 1 de octubre de 2013. Disponible en: http://www.aimc.es/-La-Television-Tradicional-vs,193-.html.

Bardoel, J. (1996). Beyond journalism, en European journal of communication, vol. 1, (3), p. 282-302.

Barredo Ibáñez, D. y Oller Alonso, M. (2012). Las fuentes de información y los enlaces hipertextuales en las coberturas informativas de los enfrentamientos entre Real Madrid y F.C.Barcelona en Marca.com y Sport.es, en Miguel Hernández Journal, 3, p. 217-234.

Bowman, S. y Willis, C. (2003). We Media: How audiences are shaping the future of news and information. Reston, Va, The Media Center at the American Press Institute. Hypergene. Consultado el 5 de octubre de 2013. Disponible en: http://www.hypergene.net/

Bruns, A. (2005). Gatewatching: collaborative online news production. New York: Peter Lang.

Cabrera González, M. Á. (2000). La prensa online. Los periódicos en la www. Barcelona: CIMS.

Cantalapiedra, M. J. (2010). Interactividad y participación ciudadana en los medios vascos en Internet, en Mediatika. 12, p. 213-256. 
Internet - periódicos digitales - interactividad - participación ciudadana - Web 2.0

Díaz-Noci, J. et al (2009). Content and message analysis of online journalism: some methodological proposals. Proceedings of the $\mathrm{V}$ International Conference, en. Comunication and Reality, 647-656.

Deuze, M. (1999). Journalism and the web. An analysis of skills and standards in an online environment, en Gazette, vol. 61, (5), p. 373-390.

Domingo, D. et al (2008). Participatory journalism practices in the media and beyond: an international comparative study of initiatives in online newspapers, en Journalism practice, vol. 2, (3), p. 326-342.

Gillmor, D (2004). We the media: grassroots journalism by the people, for the people. Sebastopol: O'Reilly, 2004. Consultado el 3 de noviembre de 2013. Disponible en: http://www.oreilly.com/catalog/wemedia/book/

Guallar, J. y Abadal, E. (2009). Evaluación de las hemerotecas de prensa digital: indicadores y ejemplos de buenas prácticas, en El profesional de la información, 18, p. 255-269.

López García, X. (2011). Rasgos de la estrategia de los cibermedios gallegos en su empeño por promover la experimentación para el cambio de modelo periodístico, en Estudios sobre el Mensaje Periodístico, vol. 17 (1), p. 81-93.

Martínez, S. (2008). La interactividad en los diarios digitales españoles (2006-2008), en Doxa, 7, p. 157-164.

Masip, P.; Díaz-Noci, J.; Domingo, D.; Micó-Sanz, J.-L.; Salaverría, R. (2010). Investigación internacional sobre ciberperiodismo: hipertexto, interactividad, multimedia y convergencia, en El profesional de la información, 19, p. 568-576.

Meso, K.; Palomo, B.; Ganzabal M. (2009). Análisis del periodismo participativo en las principales cadenas de televisión en la era de Internet. Los casos de antena 3.com, telecinco.es, plus.es, lasexta.com, cuatro.com y rtve.es en B. León. Telerrealidad. El mundo tras el cristal. (pp. 192-204) Sevilla: Comunicación Social.

Navarro, L. (2009). Tres lustros del periodismo digital: interactividad e hipertextualidad, en Comunicar, 33, p. 35-43.

Observatorio Nacional de las Telecomunicaciones y de la SI (2011). Estudio sobre el conocimiento y uso de las Redes Sociales en España. Consultado el 6 de octubre de 2013. Disponible en: http://www.slideshare.net/retelur/redes-sociales-en-internetontsi-dic11. 
Orihuela, J. L. (2002). Los 10 paradigmas de la e-comunicación. Consultado el 5 de octubre de 2013. Disponible en: http://www.agetec.org/ageteca/Los\%2010 Paradigmas \%20 de\%20la\% 20e- Comunicacion.pdf

Palacios, M. y Díaz-Noci, J. (eds.) (2009). Online Journalism: Research Methods: A multidisciplinary approach in comparative perspective. Bilbao: Servicio Editorial de la Universidad del País Vasco.

Paterson, C. y Domingo, D. (2008). Making online news: The etnography of new media production. New York: Peter Lang.

Peña, P. (2012). Interactividad y redes sociales en la radio española, en Telos, 92, p. 105-117.

Rodríguez-Martínez, R.; Codina, L. y Pedraza-Jiménez, R. (2010). Cibermedios y web 2.0: modelo de análisis y resultado de aplicación, en El profesional de la información, 19, p. 35-44.

Rodríguez-Martínez, R.; Codina, L. y Pedraza-Jiménez, R. (2012). Indicadores para la evaluación de la calidad en cibermedios: análisis de la interacción y de la adopción de la Web 2.0, en Revista Española de Documentación Científica, 35, p. 61-93.

Salaverría, R. et al. (2005). Análisis comparativo de cibermedios: Lavanguardia.es, Elmundo.es y Elpaís.es. R. Salaverría, (coord.) Cibermedios. El impacto de internet en los medios de comunicación en España. (pp. 125-143) Sevilla: Comunicación Social Ediciones y Publicaciones.

Salaverría, R.; Díaz Noci, J.; Cores, R.; Meso, K.; Larrondo, A. (2004). Evaluación de los ciberdiarios en las comunidades vasca y navarra, en Revista Comunicación y Sociedad, vol. 17, (1), p. 161-189.

Salaverría, R.; García-Avilés, J. A.; Masip, P. (2010). Concepto de convergencia periodística. En X. López-García y X. Pereira-Fariña (coords.). Convergencia digital. Reconfiguración de los medios de comunicación en España. (pp. 41-63) Santiago de Compostela: Servicio Editorial de la Universidad de Santiago de Compostela.

Thurman, N. y Lupton, B. (2009). Convergence calls: Multimedia storytelling at British news websites, en Convergence: the international journal of research into new media technologies, 14, 4, 439-455. Consultado el 30 de octubre de 2013. Disponible en: http://con.sagepub.com/content/14/4/439.full. pdf+html

Tremayne, M.; Weiss, A. S. y Alves, R. C. (2007). From product to service: The diffusion of dynamic content in online newspapers, en Journalism $\mathcal{E}$ Mass Communication Quaterly, 84, p. 825-839. 
Internet - periódicos digitales - interactividad - participación ciudadana - Web 2.0

Vujnovic, M. et al. (2010) Exploring the political-economic factors of participatory journalism, en Journalism Practice, vol. 4 (3), p. 285-296.

Zamith, F. (2008). Ciberjornalismo: As potencialidades da Internet nos sites noticiosos portugueses. Porto: Afrontamento. 